\title{
Localization of a Bose-Einstein condensate in a bichromatic optical lattice
}

\author{
S. K. Adhikari ${ }^{1, *}$ and L. Salasnich ${ }^{2, \dagger}$ \\ ${ }^{1}$ Instituto de Física Teórica, UNESP-São Paulo State University, Barra Funda, 01.140-070 São Paulo, SP, Brazil \\ ${ }^{2}$ CNR-INFM and CNISM, Unità di Padova, Dipartimento di Fisica "Galileo Galilei,"Università di Padova, Via Marzolo 8 , \\ 35131 Padova, Italy \\ (Received 12 May 2009; published 11 August 2009)
}

\begin{abstract}
By direct numerical simulation of the time-dependent Gross-Pitaevskii equation, we study different aspects of the localization of a noninteracting ideal Bose-Einstein condensate (BEC) in a one-dimensional bichromatic quasiperiodic optical-lattice potential. Such a quasiperiodic potential, used in a recent experiment on the localization of a BEC [Roati et al., Nature (London) 453, 895 (2008)], can be formed by the superposition of two standing-wave polarized laser beams with different wavelengths. We investigate the effect of the variation of optical amplitudes and wavelengths on the localization of a noninteracting BEC. We also simulate the nonlinear dynamics when a harmonically trapped BEC is suddenly released into a quasiperiodic potential, as done experimentally in a laser speckle potential [Billy et al., Nature (London) 453, 891 (2008)]. We finally study the destruction of the localization in an interacting BEC due to the repulsion generated by a positive scattering length between the bosonic atoms.
\end{abstract}

DOI: $10.1103 /$ PhysRevA.80.023606

PACS number(s): 03.75.Nt, 03.75.Lm, 64.60.Cn, 67.85.Hj

\section{INTRODUCTION}

The localization of the electronic wave function in a disordered potential was predicted by Anderson 50 years ago [1]. More recently, the phenomenon of localization due to disorder was experimentally observed in electromagnetic waves [2,3], in sound waves [4], and also in quantum matter waves [5-8]. In the case of quantum matter waves, Billy $e t$ al. [5] observed exponential localization of a Bose-Einstein condensate (BEC) of ${ }^{87} \mathrm{Rb}$ atoms released into a onedimensional (1D) waveguide in the presence of a controlled disorder created by a laser speckle. Roati et al. [6] observed localization of a noninteracting $\mathrm{BEC}$ of ${ }^{39} \mathrm{~K}$ atoms in a $1 \mathrm{D}$ potential created by two optical-lattice (OL) potentials with different amplitudes and wavelengths. The noninteracting BEC of ${ }^{39} \mathrm{~K}$ atoms was created [6] by tuning the interatomic scattering length to zero near a Feshbach resonance [9].

In this paper, with intensive numerical simulations of the linear Gross-Pitaevskii (GP) equation (which is just the Schrödinger equation,), we study different aspects of localization of the BEC in a 1D bichromatic quasiperiodic OL potential used in the experiment of Roati et al. [6]. The 1D quasiperiodic potentials have a spatial ordering that is intermediate between periodicity and disorder [10-12]. In particular, the 1D discrete Aubry-Andre model of quasiperiodic confinement $[11,12]$ displays a transition from extended to localized states which resembles the Anderson localization of random systems $[13,14]$. Modugno [15] recently showed that the linear 1D Schrödinger equation with a bichromatic periodic potential can be mapped in the Aubry-Andre model and he studied the transition to localization as a function of the parameters of the periodic potential.

To investigate the interplay between the bichromatic potential and the interatomic interaction in the localization of a

\footnotetext{
*adhikari@ift.unesp.br; www.ift.unesp.br/users/adhikari

†luca.salasnich@pd.infn.it; www.padova.infm.it/salasnich
}

BEC, we adopt the 1D nonlinear GP equation [16,17] in place of the linear 1D Schrödinger equation used to describe a noninteracting BEC. We find that the nonlinearity of the GP equation, which accurately models the binary interatomic interaction of atoms, has a strong effect on localization and a reasonably weak repulsive nonlinear term is capable of destroying the localization. Our results on the effect of nonlinearity in the localization are thus in qualitative agreement with similar predictions based on the 1D discrete nonlinear Schrödinger equation (DNLSE) with random on-site energies [18]. First effects of a weak nonlinearity in Anderson localization have been shown experimentally in light waves in photonic crystals $[6,19]$.

There have already been a number of theoretical studies on Anderson localization under the action of different potentials. Sanchez-Palencia et al. and Clément et al. considered Anderson localization in a random potential [13]. Damski et $a l$. and Schulte et al. considered Anderson localization in disordered OL potential [14]. There have been studies of Anderson localization with other types of disorder [20]. Effect of interaction on Anderson localization was also studied [21]. Anderson localization in BEC under the action of a disordered potential in two and three dimensions has also been investigated [22]. In this paper, we study different aspects of the localization of an ideal BEC and the delocalization of an interacting BEC in a quasiperiodic OL potential using the linear and nonlinear 1D GP equations.

In Sec. II, we present a brief account of the nonlinear 1D GP equation used in our study and of the variational solution of the same under appropriate conditions. In Sec. III, we present our numerical studies on localization using time propagation under the Crank-Nicolson discretization scheme. The density profiles of the tightly localized states are in agreement with the variational results. We also study the effects of the variation of the wavelength and intensity of the OL potentials on the localization. We study the nonequilibrium dynamics, as observed in the experiment of Billy et al. [5], when a harmonically trapped BEC is suddenly released from the harmonic trap into a quasiperiodic OL potential. We 
also investigate the destruction of localization in the presence of a repulsive atomic interaction and it is found that a reasonably small nonlinearity can destroy the localization of the BEC. In Sec. IV, we present a brief discussion and concluding remarks.

\section{ANALYTICAL CONSIDERATION OF LOCALIZATION}

In the actual experiment of Roati et al. [6], the 1D quasiperiodic bichromatic OL potential was produced by superposing two OL potentials generated by two standing-wave polarized laser beams of slightly different wavelengths and amplitudes, which we take here similar to those in the experiment of Roati et al., e.g., with wavelengths $\hat{\lambda}_{1}$ $=1032 \mathrm{~nm}$ and $\hat{\lambda}_{2}=862 \mathrm{~nm}$. This $1 \mathrm{D}$ quasiperiodic OL potential can be written as [6]

$$
V(\hat{z})=\sum_{i=1}^{2} 2 s_{i} E_{i} \cos ^{2}\left(k_{i} \hat{z}\right),
$$

where $2 s_{i}, i=1,2$, are the amplitudes of the OL potentials in units of respective recoil energies $E_{i}=2 \pi^{2} \hbar^{2} /\left(m \hat{\lambda}_{i}^{2}\right), \quad k_{i}$ $=2 \pi / \lambda_{i}, i=1,2$, are the respective wave numbers, $\hbar$ is the reduced Planck constant, and $m$ the mass of an atom.

With a single periodic potential of the form $\cos ^{2}(k \hat{z})$ with $s_{2}=0$, the linear Schrödinger equation permits only delocalized states in the form of Bloch waves. Localization is possible in the linear Schrödinger equation due to the "disorder" introduced through a second component in Eq. (1). The localization is not intuitively obvious. The potential (1) continues to have an infinite number of finite barriers as in a simple OL potential and it might be expected that any localized state will decay due to tunneling.

The localized states that we study are low-lying states of the system with potential (1). They are quite distinct from the so-called gap solitons in a simple OL potential appearing for repulsive nonlinearity in the band gap of the spectrum of the linear Schrödinger equation [23]. These gap solitons with finite spatial extension are excited states of the system without a linear counterpart.

The dynamics of a trapped BEC of $N$ atoms in a transverse harmonic potential of frequency $\omega_{\perp}$ plus the axial quasiperiodic OL potential (1) is determined by the following GP equation $[16,17]$ :

$$
i \frac{\partial}{\partial t} \psi(\mathbf{r}, t)=\left[-\frac{\nabla^{2}}{2}+\frac{x^{2}+y^{2}}{2}+V(z)+2 \pi g|\psi(\mathbf{r}, t)|^{2}\right] \psi(r, t),
$$

$$
V(z)=\sum_{i=1}^{2} \frac{4 \pi^{2} s_{i}}{\lambda_{i}^{2}} \cos ^{2}\left(\frac{2 \pi}{\lambda_{i}} z\right),
$$

with normalization $\int|\psi(\mathbf{r}, t)|^{2} d \mathbf{r}=1$ and where $g=2 N \hat{a} / a_{\perp}$ is the dimensionless interaction strength, with $\hat{a}$ the interatomic scattering length and $a_{\perp}=\sqrt{\hbar /\left(m \omega_{\perp}\right)}$ the characteristic harmonic length of the transverse confinement, and $\mathbf{r} \equiv(x, y, z)$ defines the Cartesian coordinates. Here, we have considered the harmonic trap $\left(x^{2}+y^{2}\right) / 2$ in transverse directions $(x, y)$ and the quasiperiodic potential $V(z)$ in the longitudinal direction $z$. In Eq. (2), length is in units of $a_{\perp}$, time in units of $\omega_{\perp}$, and energy in units of $\hbar \omega_{\perp}$. In the noninteracting case, $g$ $=0$ and Eq. (2) becomes the usual linear Schrödinger equation.

Another completely equivalent potential is the one where the cosine term of Eq. (3) is replaced by a sine

$$
V(z)=\sum_{i=1}^{2} \frac{4 \pi^{2} s_{i}}{\lambda_{i}^{2}} \sin ^{2}\left(\frac{2 \pi}{\lambda_{i}} z\right)
$$

However, potential (4) generates a different type of localized states compared to potential (3). Potential (4) has a local minimum at the center $z=0$, consequently, stable stationary solutions with this potential have a maximum at $z=0$. However, potential (3) has a local maximum at $z=0$ corresponding to a minimum of the stationary solution at the center. We shall show that, starting with an initial Gaussian wave function centered at $z=0$, the numerical solution of the GP Eq. (2) gives different localized eigenstates depending on the choice of the confining potential.

For a cigar-shaped trap with strong transverse confinement, it is appropriate to consider a $1 \mathrm{D}$ reduction of Eq. (2) by freezing the transverse dynamics to the respective ground state and integrating over the transverse variables. The resulting three-dimensional (3D)-1D reduction of Eq. (2) for small nonlinearity $g$ is realized via $[24,25]$

$$
i \frac{\partial}{\partial t} \phi(z, t)=\left[-\frac{\partial_{z}^{2}}{2}+V(z)+g|\phi(z, t)|^{2}\right] \phi(z, t),
$$

with normalization $\int_{-\infty}^{\infty} d z|\phi(z, t)|^{2}=1$ and where $\phi(z, t)$ is the axial wave function of the Bose condensate. Equation (5) is a 1D nonlinear Schrödinger equation with cubic nonlinearity.

Modugno used potential (4) in his study and in addition took $\lambda_{1}=2 \pi$ and defined $s_{i}^{\prime}=2 s_{i}$ and $\beta=\lambda_{1} / \lambda_{2} \equiv 2 \pi / \lambda_{2}$, so that [viz. Eq. (16) of Ref. [15]]

$$
V(z)=\frac{1}{2} s_{1}^{\prime} \sin ^{2}(z)+\frac{1}{2} s_{2}^{\prime} \beta^{2} \sin ^{2}(\beta z) .
$$

Equation (5) with potential (6) can be mapped [15] in a nonlinear version of the Aubry-Andre model $[10,11]$ by expanding the wave function $\phi(z, t)=\Sigma_{j} c_{j}(t) W_{j}(z)$ over a set of orthonormal Wannier states $W_{j}(z)$, where $W_{j}(z)$ is maximally localized at the $j$ th minimum of the primary lattice. In this way, one finds that the complex coefficients $c_{j}(t)$ satisfy the time-dependent discrete nonlinear Schrödinger equation [15]

$$
\begin{aligned}
i \frac{d}{d t} c_{j}(t)= & -J\left[c_{j+1}(t)+c_{j-1}(t)\right]+\Delta \cos (2 \pi \beta j) c_{j}(t) \\
& +\widetilde{g}\left|c_{j}(t)\right|^{2} c_{j}(t),
\end{aligned}
$$

where $\left|c_{j}(t)\right|^{2}$ gives the probability of occupation of the $j$ th site at time $t$. The hopping term $J$ and the "disorder" term $\Delta$ are connected to the parameters of the bichromatic potential (1) and can be calculated by using the Wannier functions $[15,26]$. Modugno [15] showed that the linear $(g=0)$ GP equation (5) displays localization for a large enough $\Delta / J$ and the localization increases as $\Delta / J$ increases for a wide range of values of $\beta$. 
The Fourier transformation $f(k)$ of the Anderson localized state $\phi(z)$ is defined as

$$
f(k)=\int_{-\infty}^{\infty} \exp (i 2 \pi k z) \phi(z),
$$

and the momentum distribution of the localized state $P(k)$ $\equiv f^{2}(k)$.

Usually, the stationary bound states formed with quasiperiodic OL potentials (3) and (4) occupy many sites of the quasiperiodic OL potential and have many local maxima and minima. For certain values of the parameters, potential (4) leads to bound states confined practically to the central site of the quasiperiodic OL potential. When this happens, a variational approximation with Gaussian ansatz leads to a reasonable prediction for the bound state.

The stationary form of the linear Schrödinger equation (5) (with $i \partial / \partial t$ replaced by a chemical potential $\mu$ ) with potential (4) can be derived from the following Lagrangian:

$$
L=\int_{-\infty}^{\infty}\left[\mu \phi^{2}(z)-\left\{\phi^{\prime}(z)\right\}^{2} / 2-V(z) \phi^{2}(z)\right] d z-\mu,
$$

by demanding $\delta L / \delta \phi=\delta L / \delta \mu=0$, where the prime denotes space derivative. To apply the variational approximation, we use the Gaussian ansatz [27]

$$
\phi(z)=\pi^{-1 / 4} \sqrt{\frac{\mathcal{N}}{w}} \exp \left(-\frac{z^{2}}{2 w^{2}}\right),
$$

where variational parameters are the norm $\mathcal{N}$, width $w$, and $\mu$. This ansatz implies that the center of the stationary state is placed at the local minimum at $z=0$ of the quasiperiodic OL potential (4). The substitution of ansatz (10) in Lagrangian (9) leads to

$$
L=\mu(\mathcal{N}-1)-\frac{\mathcal{N}}{4 w^{2}}+\sum_{i=1}^{2} \frac{A_{i} \mathcal{N}}{2}\left[\exp \left(-\alpha_{i}^{2} w^{2}\right)-1\right],
$$

where $A_{i}=4 \pi^{2} s_{i} / \lambda_{i}^{2}, \alpha_{i}=2 \pi / \lambda_{i}$. The first variational equation from Eq. (11), $\partial L / \partial \mu=0$, yields $\mathcal{N}=1$, which will be used in other variational equations. The second variational equation $\partial L / \partial w=0$ yields

$$
1=\sum_{i=1}^{2} 2 \alpha_{i}^{2} A_{i} w^{4} \exp \left(-\alpha_{i}^{2} w^{2}\right)
$$

and determines the width $w$. The last variational equation $\partial L / \partial \mathcal{N}=0$ yields

$$
\mu=1 /\left(4 w^{2}\right)-\sum_{i=1}^{2} A_{i}\left[\exp \left(-\alpha_{i}^{2} w^{2}\right)-1\right] / 2,
$$

which defines the chemical potential. (However, some caution should be exercised in using these variational equations: they predict a false bound state for a one-term periodic potential with $s_{2}=0$ [cf. Eq. (12)] and the one-term potential is known to support no localized bound state.)

\section{NUMERICAL RESULTS}

To perform a systematic numerical study of localization with potentials (3) and (4), we vary $\lambda_{1}$ and $\lambda_{2}$ maintaining the ratio $\lambda_{2} / \lambda_{1}=0.86$ (roughly the same ratio $\lambda_{2} / \lambda_{1}$ as in the experiment of Roati et al. [6]). We consider a transverse harmonic-oscillator length $a_{\perp} \approx 1 \mu \mathrm{m}$ so that the experimental wavelengths $\hat{\lambda}_{1}=1032 \mathrm{~nm}$ and $\hat{\lambda}_{2}=862 \mathrm{~nm}$ in dimensionless units become $\lambda_{1} \approx 1$ and $\lambda_{2} \approx 0.86$. In the first part of the study, we also set scattering length $\hat{a}=0$ corresponding to ideal noninteracting bosons.

We perform the numerical simulation employing mostly real-time propagation with Crank-Nicholson discretization scheme, using the FORTRAN programs provided in Ref. [28], with space step 0.025 and time step 0.0005 . Imaginary-time propagation routine can determine the strongly localized state confined to a single OL site or so in an efficient fashion. However, imaginary-time propagation routine demonstrated difficulty for weakly confined state extending over a large number of OL sites.

Because of the oscillating nature of the potential, great care was needed to obtain a precise localized state. The accuracy of the numerical simulation was tested by varying the space and time steps as well as the total number of space steps. A larger value of the ratio $s_{2} / s_{1}$ gives more binding for the localized state. Consequently, the localized state has smaller spatial extension.

To understand the nature of these localized states and their behavior under the variation of different parameters, first we consider the localized states with larger values of $\lambda_{1}$. Such states with large $s_{2} / s_{1}(=1)$ occupy a small number of OL sites and hence their simulation can be performed relatively easily. The shape of the localized state then becomes a quasiGaussian for potential (4) and we compare our numerical results to the variational results in this case. In Fig. 1(a), we plot the results of density distribution from numerical and variational calculations for potential (4) for different $\lambda_{1}$ for fixed $s_{1}, s_{2}$ and $\lambda_{2} / \lambda_{1}$. In Fig. 1(a), real- and imaginary-time propagation routines produced identical results. In Fig. 1(b), we plot the density distribution $\phi^{2}(z)$ vs $z$ for potential (3) for the same set of parameters as in Fig. 1(a). In this case, one has two peaks in the density distribution. We also calculated the energies of these states. The energies for $\lambda_{1}=15,10$, and 5 for the potential (4) of Fig. 1(a) are 0.264, 0.599, and 2.374 to be compared to the variational results of Eq. (13): $0.266,0.594$, and 2.396 , respectively. This agreement between the numerical and variational results of density for potential (4) in Fig. 1(a) and of the respective energies provides assurance about the accuracy of the numerical code used in simulation in our investigation. We also calculated the (numerical) energies of the density profiles displayed in Fig. 1(b) which are, respectively, 0.270, 0.603, and 2.412. These energies for potential (3) are distinct from those of potential (4); but the two sets of energies are very close to each other.

Now we present the results for the solution of Eq. (5) with potential (3) for a small $s_{2} / s_{1}$. The potential $V(z)$ given by Eq. (3) for $\lambda_{1}=2, \lambda_{2} / \lambda_{1}=0.86, s_{1}=2$, and $s_{2} / s_{1}=0.2$ is plotted in Fig. 2(a). This potential is quite similar to the potential 

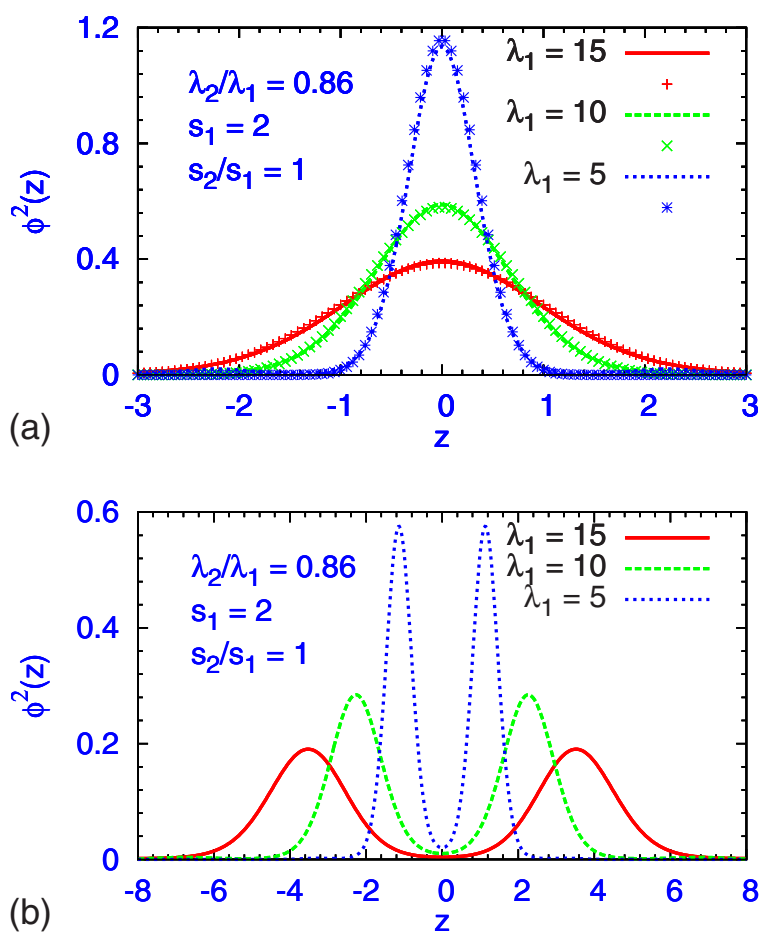

FIG. 1. (Color online) (a) Typical density distribution $\phi^{2}(z)$ vs $z$ for a noninteracting BEC for potential (4) for $\lambda_{2} / \lambda_{1}=0.86, s_{1}=s_{2}$ $=2$, and $\lambda_{1}=15,10$, and 5 . The numerical results are shown by continuous lines. The variational results are shown by a chain of symbols. (b) Typical density distribution $\phi^{2}(z)$ vs $z$ for potential (3) for same parameters as in (a). Both $\phi^{2}(z)$ and $z$ are in dimensionless units.

illustrated in Fig. 1(a) of Roati et al. [6]. The density distribution $\phi^{2}(z)$ vs $z$ corresponding to the localization for this potential is plotted in Fig. 2(b) for $s_{1}=2, s_{2} / s_{1}=0.2, \lambda_{2} / \lambda_{1}$ $=0.86$, and $\lambda_{1}=2,4,8$, and 12 . It is seen that with a decrease of $\lambda_{1}$, more attraction is created. Consequently, the states with $\lambda_{1}=2$ and 4 are more localized in space with lesser spatial extension. But with further increase of $\lambda_{1}$, a single site of the quasiperiodic OL potential occupies a large region in space. When this happens, the spatial size of the localized bound states increases with $\lambda_{1}$, as the localized state cannot occupy less than two sites of the quasiperiodic OL potential [the density for potential (3) has to be symmetric around $z$ $=0$ and must have a minimum at $z=0]$. This happens for $\lambda_{1}=8$ and 12. For $\lambda_{1}=8$ and 12, the localized bound states occupy four sites of the quasiperiodic OL potential. Partially similar feature is also exhibited by the localized states of the quasiperiodic OL potential (4) for larger values of $\lambda_{1}$ as shown in Fig. 2(c), where we plot the density distribution $\phi^{2}(z)$ vs $z$ for $s_{2}=2, s_{2} / s_{1}=0.2, \lambda_{2} / \lambda_{1}=0.86$, and $\lambda_{1}=2,4,8$, and 12. As expected, the localized state with potential (3) has a minimum at $z=0$, whereas the localized state of potential (4) has a maximum at $z=0$. We plot in Figs. 3(a) and 3(b) the momentum distribution $P(k)$ vs $k$ for the localized states-a quantity of experimental interest [6] - shown in Figs. 3(a) and 3(b) for potentials (3) and (4), respectively. In general, as expected, the central peak of momentum distribution of the localized state is sharper for a localized state of larger spatial extension.
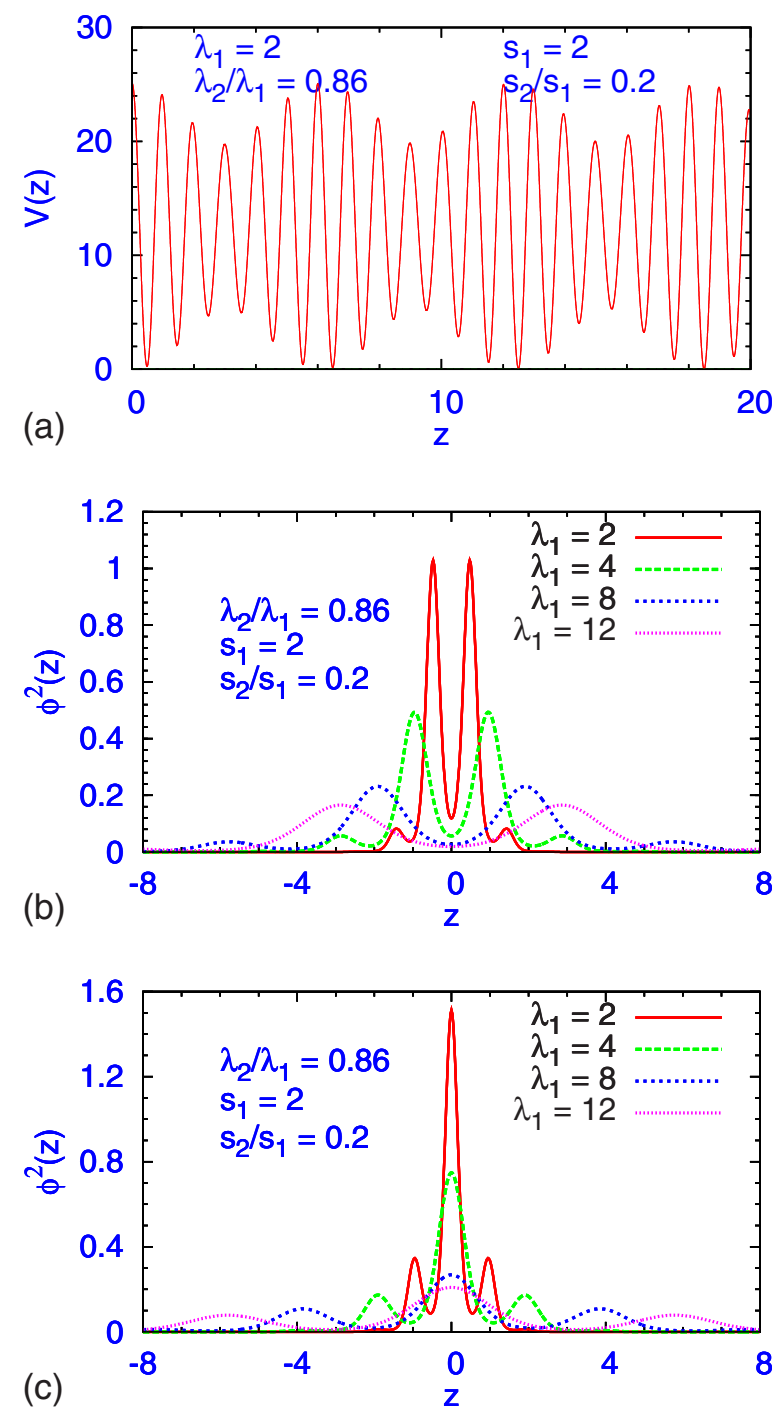

FIG. 2. (Color online) (a) Potential $V(z)$ vs $z$ given by Eq. (3) for $\lambda_{1}=2, \lambda_{2} / \lambda_{1}=0.86, s_{1}=2$, and $s_{2} / s_{1}=0.2$ and (b) density distribution $\phi^{2}(z)$ vs $z$ of a noninteracting BEC calculated with this potential for $s_{1}=2, s_{2} / s_{1}=0.2, \lambda_{2} / \lambda_{1}=0.86$, and $\lambda_{1}=2,4,8$, and 12 . (c) Density distribution $\phi^{2}(z)$ vs $z$ of a noninteracting BEC for potential (4) for $s_{1}=2, s_{2} / s_{1}=0.2, \lambda_{2} / \lambda_{1}=0.86$, and $\lambda_{1}=2,4,8$, and 12. All variables are expressed in dimensionless units.

Now we study how these localized states are affected by a variation of the ratio $s_{2} / s_{1}$ when $\lambda_{1}, \lambda_{2}$, and $s_{1}$ are maintained constant. For this purpose, we plot in Figs. 4(a) and 4(b) the density distribution of the localized states for potentials (4) and (3), respectively, for $\lambda_{1}=10, \lambda_{2}=8.6, s_{1}=2$, and for different values of the fraction $s_{2} / s_{1}=0.2,0.4$, and 1 . In addition, we plot the quasiperiodic OL potential $V(z)$ for $s_{2} / s_{1}=0.2$ in arbitrary units just to compare the position of the maxima and minima of the potential to the position of the minima and maxima of density. [The positions of the maxima and minima of the potential $V(z)$ do not change visibly with $s_{2} / s_{1}$.] It is found in both cases that the states with larger values of the fraction $s_{2} / s_{1}$ have smaller spatial extension corresponding to larger attraction. The results reported in Figs. 4(a) and 4(b) are in qualitative agreement with a conclusion of the study of Modugno [15] that the 

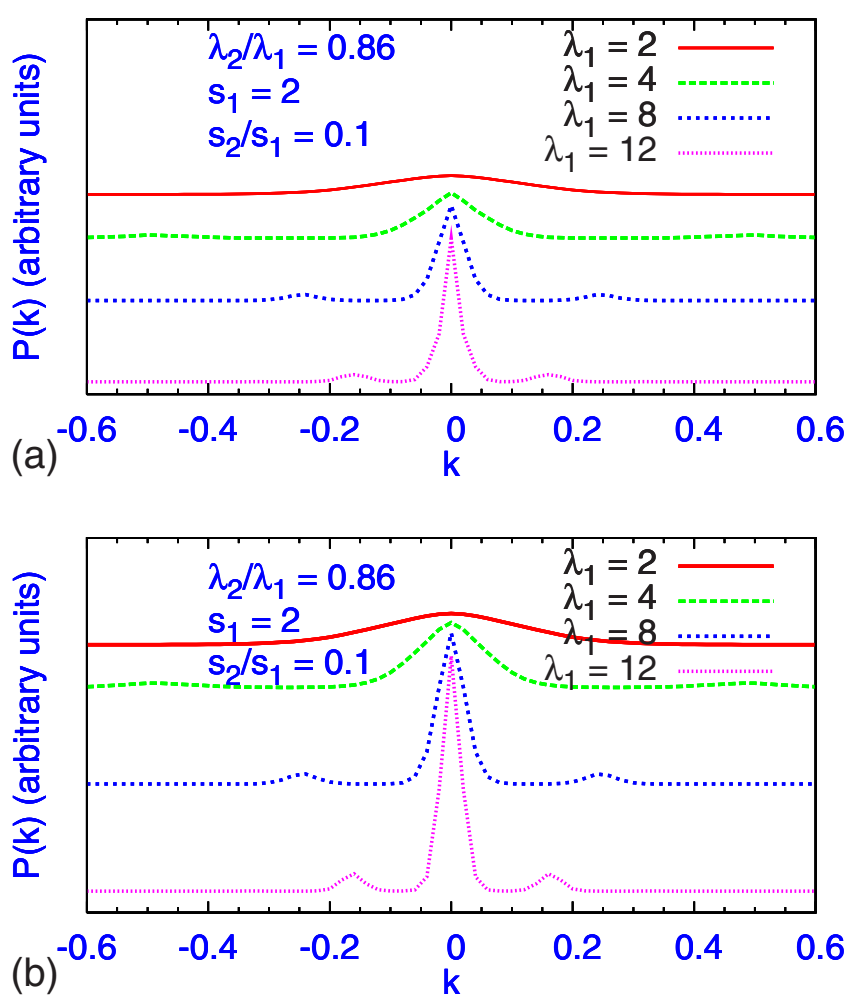

FIG. 3. (Color online) Momentum distribution $P(k)$ vs $k$ of the localized states, shown in Figs. 2(b) and 2(c), for (a) potential (3) and (b) potential (4), for $\lambda_{2} / \lambda_{1}=0.86, s_{1}=2, s_{2} / s_{1}=0.2$, and $\lambda_{1}=2$, 4,8 , and 12 .

localization appears and increases as the disorder to hopping ratio $\Delta / J$ increases. The increase of $\Delta / J$ is related to an increase of $s_{2} / s_{1}$ for a fixed $\lambda_{1}$ and $\lambda_{2}$, exactly as illustrated in Figs. 4(a) and 4(b). However, because of the distinct model (DNLSE) used in Ref. [15], in contrast to the numerical solution of the GP equation in the present study, a quantitative comparison of the results of the two studies is not to the point.

Next, we study the effect of including interaction in a BEC of ${ }^{39} \mathrm{~K}$ atoms with scattering length $\hat{a}=33 a_{0}$ $=1.75 \mathrm{~nm}$ [29] (with $a_{0}=05292 \mathrm{~nm}$, the Bohr radius) by solving Eq. (5) with potentials (3) and (4). In present dimensionless units, this will correspond to a scattering length of $a \equiv \hat{a} / a_{\perp}=0.00175$. The inclusion of the repulsive nonlinear potential term in Eq. (5) will reduce the possibility of the appearance of localized bound states. This is illustrated in Figs. 5(a) and 5(b) where we plot the density distribution for $\lambda_{1}=4, \lambda_{2} / \lambda_{1}=0.86, s_{1}=2$, and $s_{2} / s_{1}=0.2$ for potentials (4) and (3), respectively, for different $g \equiv 2 N \hat{a} / a_{\perp}=(0,2,4,5)$. The corresponding quasiperiodic OL potentials are also plotted in arbitrary units. (The advantage of using the variable $g$, rather than the scattering length $\hat{a}$ and number of atoms $N$, in these plots is that the present plots can easily be used to simulate different experimental situations with different traps and distinct bosonic atoms.) For $g=0$, for both potentials, the localized states are confined between $z= \pm 10$. For $g=2$, with increased repulsion, the matter density is reduced in the central peaks and new peaks appear for larger $z$ values. For $g$ $=4$, with further increase in repulsion, the matter density is
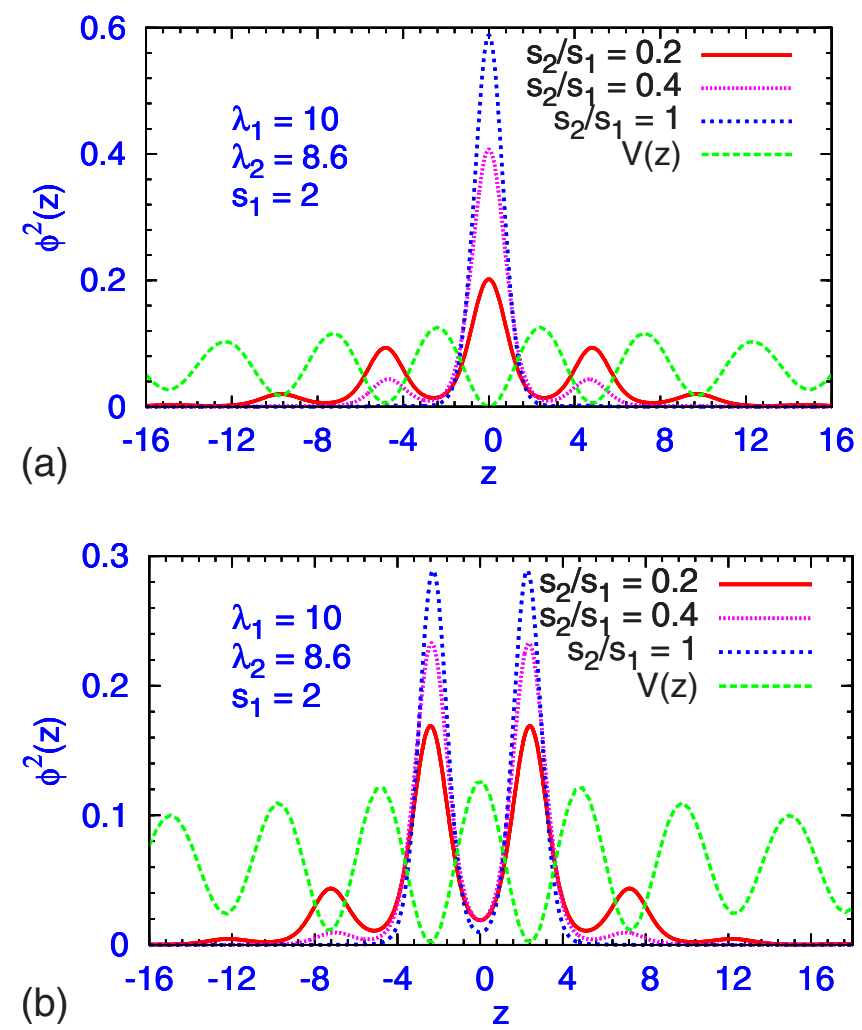

FIG. 4. (Color online) Typical density distribution $\phi^{2}(z)$ vs $z$ for a noninteracting BEC for (a) potential (4) and (b) potential (3) for $\lambda_{1}=10, \lambda_{2}=8.6, s_{1}=2$, and $s_{2} / s_{1}=0.2,0.4$, and 1 . The quasiperiodic OL potential $V(z)$ for $s_{2} / s_{1}=0.2$ is also plotted in (a) and (b) in arbitrary units. All variables are in dimensionless units.

further reduced in the central region and new peaks appear in the form of undulating tails near the edges. With further increase in the value of $g$, the localized states have larger and larger spatial extension and soon the nonlinear repulsion is so large that no localized states are possible and this happens rapidly as $g$ is increased beyond 6. The nonlinearity in Eq. (5) is $g=2 \hat{a} N / a_{\perp}$ and for about $1800{ }^{39} \mathrm{~K}$ atoms with $a$ $=0.00175[29]$, the nonlinearity has the typical numerical value $g \approx 6$. Such a small nonlinearity can have a large effect on localization of a ${ }^{39} \mathrm{~K}$ BEC and can prohibit the localization. However, the number of $\mathrm{K}$ atoms can be proportionately increased if the scattering length is reduced by varying an external background magnetic field near a Feshbach resonance [9]. As $g$ value is increased, the root mean square (rms) size of the BEC increases before reaching a critical $g$ value $(>6)$ corresponding to the destruction of localization. (It is difficult to obtain accurately the critical value of $g$ needed to destroy the localization.) The increase in the rms size of the localized state with the increase in $g$ is illustrated in Fig. 5(c) where we plot the rms size vs $g$ for potentials (3) and (4). It should be noted that in the experiment of Roati $e t$ al. [6], the residual scattering length of ${ }^{39} \mathrm{~K}$ atoms near the Feshbach resonance was $0.1 a_{0}(=0.0053 \mathrm{~nm})$, e.g., they can vary the scattering length in such small steps. Thus, it should be possible experimentally to obtain the curves illustrated in Fig. 5(c) and compare them to the present investigation.

So far, we studied the stationary properties of the localized state. Next we study some dynamical aspects of the 


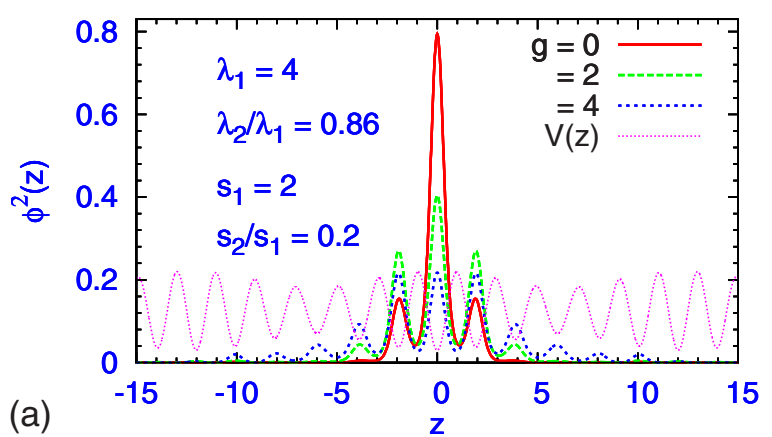

(a)
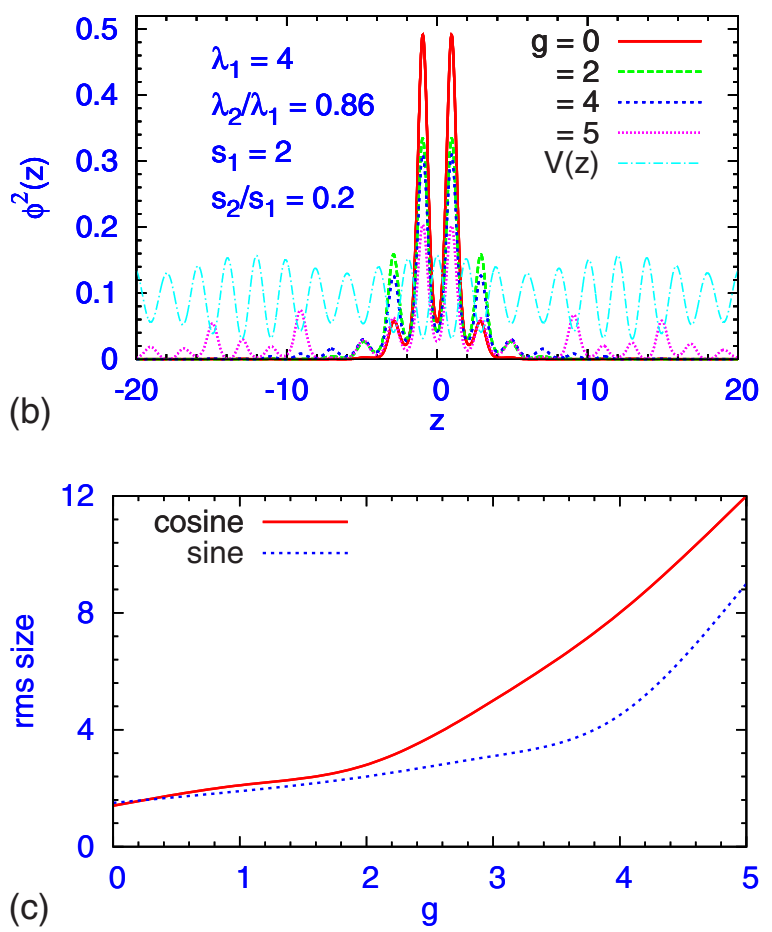

FIG. 5. (Color online) Typical density distribution $\phi^{2}(z)$ vs $z$ for an interacting BEC for different $g \equiv 2 N \hat{a} / a_{\perp}$ for (a) potential (4) and (b) potential (3) for $\lambda_{1}=4, \lambda_{2} / \lambda_{1}=0.86, s_{1}=2$, and $s_{2} / s_{1}=0.2$. The quasiperiodic OL potential $V(z)$ is plotted in arbitrary units. (c) The rms size vs nonlinearity $g$ of the stable condensate in the quasiperiodic potentials (3) (cosine) and (4) (sine) for $\lambda_{1}=4, \lambda_{2} / \lambda_{1}$ $=0.86, s_{1}=2$, and $s_{2} / s_{1}=0.2$. All quantities plotted are in dimensionless units.

localization. One interesting feature is what happens when a $\mathrm{BEC}$ is released from a harmonic trap into a quasiperiodic OL trap as investigated in the experiment of Billy et al. [5]. Mathematically, it means that at time $t=0$, the harmonic trap is suddenly changed into a quasiperiodic OL trap. The evolution of the rms size of the BEC with time is plotted in Fig. 6(a) for potential (4). The rms size first increases with the expansion of the BEC and after a certain amount of expansion, it will be locked in an appropriate localized state. After this happens, the system executes breathing oscillation around a mean shape of the localized state and the rms size remains bounded and does not increase indefinitely with time. To compare to this behavior, we also plotted the rms size for expansion in a pure periodic OL potential when the disorder is removed by setting $s_{2}=0$ in potential (4). In that

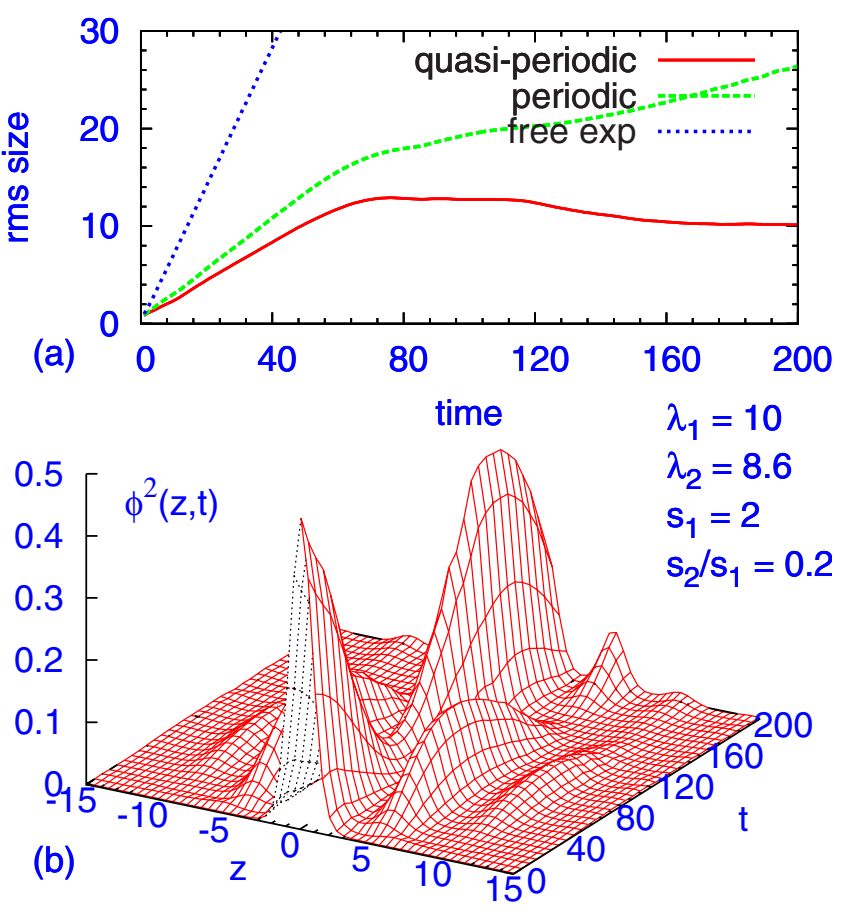

FIG. 6. (Color online) (a) The rms size of an expanding BEC released from a harmonic trap into the quasiperiodic OL potential (4) with $\lambda_{1}=10, \lambda_{2}=8.6, s_{1}=2$, and $s_{2} / s_{1}=0.2$ at time $t=0$. The rms sizes during expansions in a periodic OL potential with $s_{2}=0$ and free expansion are also shown. (b) The density profiles during above expansion in the quasiperiodic OL potential are illustrated by plotting $\phi^{2}(z, t)$ vs $z$ and $t$.

case, there is no localized state and the system expands forever with an ever-increasing rms size. Finally, when all OL potentials are removed by setting $s_{1}=s_{2}=0$, the system increases monotonically with a higher expansion rate. The dynamical density profile of the BEC during the expansion and locking in a localized state for the quasiperiodic potential (4) is shown in Fig. 6(b) where the initial expansion until $t=80$ and the consequent breathing oscillation of the BEC are clearly illustrated.

\section{CONCLUSION}

In this paper, using the numerical solution of the GP equation, we studied the localization of a noninteracting BEC in a quasiperiodic 1D OL potential prepared by two overlapping polarized standing-wave laser beams with different wavelengths and amplitudes. Specifically, we considered two analytical forms (sine and cosine) of the OL potential. We studied the effect of the variation of wavelengths and amplitudes on the localization. We also studied the nonlinear dynamics when a BEC is released from a $1 \mathrm{D}$ harmonic trap into a quasiperiodic 1D OL trap. After release, the BEC first expands (from the tightly bound harmonic-oscillator bound state) and then the expansion is stopped and the BEC is found to be trapped into one of the localized states in the quasiperiodic OL potential, as observed by Billy et al. [5].

We also studied the effect of a repulsive atomic interaction on localization. It is found that a repulsive atomic inter- 
action destroys the localization. We studied the route to this destruction of localization in some details. In particular, we investigated the localization as the nonlinearity $g \equiv 2 \mathrm{Na} / a_{\perp}$ of the nonlinear 1D GP equation is increased. It is found that as $g$ is gradually increased, the localization is slowly weakened with the localized state extending over a large space domain. Eventually, for $g$ greater than about 6 or so, the localization is destroyed.

There have been previous studies of some aspects of the localization of a BEC in a quasiperiodic potential [15] and also its destruction [18]. (It should be noted that the present study is mostly complimentary to these previous studies rather than overlapping.) However, in the present study, we consider a direct numerical solution of the GP equation as opposed to a solution of the DNLSE used in the previous studies. In view of the rapidly oscillating nature of the quasiperiodic OL potential and of the solution of the nonlinear equation, the results of the direct numerical solution of the GP equation, as used in the present investigation, should be more useful for a direct comparison to the experiments.

Note added in proof. Recently, we became aware of similar works [30].

\section{ACKNOWLEDGMENTS}

FAPESP (Brazil), CNPq (Brazil), and CARIPARO (Italy) provided partial support.
[1] P. W. Anderson, Phys. Rev. 109, 1492 (1958).

[2] D. S. Wiersma et al., Nature (London) 390, 671 (1997); F. Scheffold et al., ibid. 398, 206 (1999).

[3] R. Dalichaouch et al., Nature (London) 354, 53 (1991); A. A. Chabanov et al., ibid. 404, 850 (2000).

[4] R. L. Weaver et al., Wave Motion 12, 129 (1990).

[5] J. Billy et al., Nature (London) 453, 891 (2008).

[6] G. Roati et al., Nature (London) 453, 895 (2008).

[7] J. Chabé, G. Lemarie, B. Gremaud, D. Delande, P. Szriftgiser, and J. C. Garreau, Phys. Rev. Lett. 101, 255702 (2008).

[8] E. E. Edwards, M. Beeler, T. Hong, and S. L. Rolston, Phys. Rev. Lett. 101, 260402 (2008).

[9] S. Inouye et al., Nature (London) 392, 151 (1998).

[10] P. G. Harper, Proc. Phys. Soc. London, Sect. A 68, 874 (1955).

[11] S. Aubry and G. Andre, Ann. Isr. Phys. Soc. 3, 133 (1980).

[12] D. J. Thouless, Phys. Rev. B 28, 4272 (1983).

[13] L. Sanchez-Palencia, D. Clement, P. Lugan, P. Bouyer, G. V. Shlyapnikov, and A. Aspect, Phys. Rev. Lett. 98, 210401 (2007); D. Clément, A. F. Varon, M. Hugbart, J. A. Retter, P. Bouyer, L. Sanchez-Palencia, D. M. Gangardt, G. V. Shlyapnikov, and A. Aspect, ibid. 95, 170409 (2005); J. E. Lye, L. Fallani, M. Modugno, D. S. Wiersma, C. Fort, and M. Inguscio, ibid. 95, 070401 (2005).

[14] B. Damski, J. Zakrzewski, L. Santos, P. Zoller, and M. Lewenstein, Phys. Rev. Lett. 91, 080403 (2003); T. Schulte, S. Drenkelforth, J. Kruse, W. Ertmer, J. Arlt, K. Sacha, J. Zakrzewski, and M. Lewenstein, ibid. 95, 170411 (2005).

[15] M. Modugno, New J. Phys. 11, 033023 (2009).

[16] L. Pitaevskii and S. Stringari, Bose Einstein Condensation (Oxford University Press, Oxford, 2003); C. J. Pethick and H. Smith, Bose Einstein Condensation in Dilute Gases (Cambridge University Press, Cambridge, England, 2002).

[17] E. P. Gross, Nuovo Cimento 20, 454 (1961); L. P. Pitaevskii, Zh. Eksp. Teor. Fiz. 40, 646 (1961) [Sov. Phys. JETP 13, 451 (1961)].

[18] A. S. Pikovsky and D. L. Shepelyansky, Phys. Rev. Lett. 100, 094101 (2008); S. Flach, D. O. Krimer, and Ch. Skokos, ibid.
102, 024101 (2009).

[19] T. Schwartz et al., Nature (London) 446, 52 (2007); Y. Lahini, A. Avidan, F. Pozzi, M. Sorel, R. Morandotti, D. N. Christodoulides, and Y. Silberberg, Phys. Rev. Lett. 100, 013906 (2008).

[20] C. Fort, L. Fallani, V. Guarrera, J. E. Lye, M. Modugno, D. S. Wiersma, and M. Inguscio, Phys. Rev. Lett. 95, 170410 (2005); N. Bilas and N. Pavloff, Eur. Phys. J. D 40, 387 (2006); D. R. Grempel et al., Phys. Rev. Lett. 49, 833 (1982); L. Fallani et al., ibid. 98, 130404 (2007); P. Lugan et al., ibid. 98, 170403 (2007).

[21] P. Lugan, D. Clement, P. Bouyer, A. Aspect, and L. SanchezPalencia, Phys. Rev. Lett. 99, 180402 (2007); J. E. Lye, L. Fallani, C. Fort, V. Guarrera, M. Modugno, D. S. Wiersma, and M. Inguscio, Phys. Rev. A 75, 061603(R) (2007).

[22] R. C. Kuhn, C. Miniatura, D. Delande, O. Sigwarth, and C. A. Muller, Phys. Rev. Lett. 95, 250403 (2005); S. E. Skipetrov, A. Minguzzi, B. A. van Tiggelen, and B. Shapiro, ibid. 100, 165301 (2008); E. Abrahams, P. W. Anderson, D. C. Licciardello, and T. V. Ramakrishnan, ibid. 42, 673 (1979).

[23] S. K. Adhikari and B. A. Malomed, Phys. Rev. A 79, 015602 (2009); 76, 043626 (2007); Europhys. Lett. 79, 50003 (2007); Physica D 238, 1402 (2009).

[24] L. Salasnich, A. Parola, and L. Reatto, Phys. Rev. A 65, 043614 (2002); 69, 045601 (2004).

[25] A. Muñoz Mateo and V. Delgado, Phys. Rev. A 77, 013617 (2008); 75, 063610 (2007); 74, 065602 (2006).

[26] D. J. Boers, B. Goedeke, D. Hinrichs, and M. Holthaus, Phys. Rev. A 75, 063404 (2007).

[27] V. M. Pérez-García, H. Michinel, J. I. Cirac, M. Lewenstein, and P. Zoller, Phys. Rev. A 56, 1424 (1997).

[28] P. Muruganandam and S. K. Adhikari, J. Phys. B 36, 2501 (2003); e-print arXiv:0904.3131.; S. K. Adhikari and P. Muruganandam, J. Phys. B 35, 2831 (2002).

[29] H. Wang et al., Phys. Rev. A 62, 052704 (2000).

[30] G. Roux et al., Phys. Rev. A 78, 023628 (2008); T. Roscilde, ibid. 77, 063605 (2008). 